\title{
New function of the CD44 gene: Lipid metabolism regulation in bovine mammary epithelial cells
}

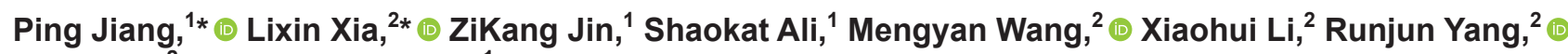 \\ Xibi Fang, ${ }^{2} \dagger$ (i) and Zhihui Zhao ${ }^{1} \dagger$ (1) \\ ${ }^{1}$ College of Agriculture, Guangdong Ocean University, Zhanjiang, 524088, P. R. China \\ ${ }^{2}$ College of Animal Science, Jilin University, Changchun, 130062, P. R. China
}

\begin{abstract}
The $C D 44$ gene encodes a cell-surface glycoprotein that participates in a variety of biological processes such as cell interactions, adhesion, hematopoiesis, and tumor metastasis. We compared the transcriptome in bovine mammary epithelial cells (bMEC) of Chinese Holstein dairy cows producing milk of high and low fat contents. Our results suggest that $C D 44$ might be a candidate gene affecting milk fat synthesis. In the present study, the overexpression of the CD 44 gene increased the contents of intracellular triglycerides (TG) and cholesterol (CHOL), whereas knockdown of the CD 44 gene decreased bMEC CHOL and TG contents. Gas chromatography analysis of fatty acid composition showed that the contents of $\alpha$-linolenic acid, palmitic acid, and cis-8,11,14-eicosatrienoic acid were altered due to changes in the level of expression of the $C D 44$ gene. Additionally, elaidic acid, palmitoleic acid, tridecanoic acid, and oleic acid were markedly reduced in the $C D 44$ gene overexpression group compared with the control group. On the contrary, cis-5,8,11,14-eicosatetraenoic acid and stearic acid were markedly increased in the CD44 knockdown group compared with the control group. And $\mathrm{RT}^{2}$ Profiler PCR array (Qiagen, CLAB24070A Frankfurt, Germany) further suggested that overexpression or knockdown of the CD44 gene altered expression levels of functional genes associated with lipid metabolism. The present data indicate that CD44 plays a key regulatory role in lipid metabolism in bMEC.
\end{abstract}

Key words: CD44, triglycerides, cholesterol, fatty acids, bovine mammary epithelial cells

\footnotetext{
Received August 6, 2019.

Accepted February 24, 2020.

*These 2 authors contributed equally to this work.

†Corresponding authors: fangxibi@jlu.edu.cn and zhzhao@jlu.edu
}

\section{INTRODUCTION}

The CD44 gene (ENSBTAG00000011578) is located on bovine chromosome 15:66,454,331-66,541,790, with 3 alternatively spliced transcripts. The transcript of CD44-201 was selected. The CD44 DNA sequence contains 87,460 base pairs with 9 exons and 8 introns. The CD 44 mRNA sequence contains a coding region of 1,107 $\mathrm{bp}$, and the open reading frame encodes 368 amino acids. The CD44 protein is a hyaluronan-binding surface receptor that is highly expressed in hematopoietic cells and immune cells (Schmits et al., 1997; Kang et al., 2013). High $C D 44$ expression is observed in early development of T- and B-cells, and upregulated expression of $C D 44$ was attributed to antigen receptor stimulation or mitogen activation (Camp et al., 1991). Fraser and colleagues observed that $C D 44$ was highly expressed in human adipogenic stem cells as a cell-surface marker (Fraser et al., 2006), which suggests that CD44 may be related to the pluripotency and differentiation of adipocyte precursors. Furthermore, previous studies have observed that cells with high expression of $C D 44$, but without the expression of CD34 or CD56, have the capacity to differentiate into adipocytes (Khan et al., 2012). Previous study has also suggested that treatment with CD44 monoclonal antibodies on mice with a high-fat diet inhibited high-fat diet-induced obesity, improved adipose tissue inflammation, and regulated the expression levels of lipid-related genes (Kodama et al., 2012). Furthermore, this effect of the antibody was superior to the effects of obesity drugs such as metformin (Kodama et al., 2012), suggesting that the CD44 gene is closely associated with lipid metabolism in mice. Additionally, our previous transcriptome analysis also revealed that $C D 44$ is a differentially expressed gene in bovine mammary epithelial cells (bMEC) of Chinese Holstein dairy cows producing milk of high and low fat contents (Supplemental Table S1, https://doi .org/10.3168/jds.2019-17415). Thus, it is possible that CD 44 might play a key role in the regulation of lipid metabolism in bMEC. 
The main components of milk fat are triglycerides (TG), which constitute about 97 to $98 \%$ of the fat content, with fatty acids (FA) and cholesterol (CHOL) as the minor components that make up around $0.1 \%$ and 0.2 to $0.4 \%$ of the fat content, respectively (Jensen et al., 1991; Lubary et al., 2011).

Although the function of $C D 44$ in lipid metabolism has been examined in mice, its effects on milk fat synthesis in dairy cattle have not been studied, especially the effects on TG and CHOL metabolism in bMEC. In our previous studies, analysis of transcriptome results suggested that $C D 44$ might be involved in fat synthesis by regulating other milk fat metabolism-related genes. Therefore, the objective of the present study was to uncover the regulatory roles of CD44 in bMEC on the contents of TG, CHOL, and FA, as well as on expression levels of genes involved in lipid and FA metabolism, using gene overexpression and RNA interference (RNAi) approaches. This study will be valuable for revealing the regulatory roles and molecular mechanisms of $C D 44$ in milk fat synthesis.

\section{MATERIALS AND METHODS}

\section{Constructing Interference Vector pb7SK-shCD44}

The target sequence of the CD44 gene (GenBank: NM_174013.3) is GCGCAGATCGATCTGAATATA. The forward primer is 5'-AGAGGCGCAGATCGATCTGAATATATCTCTTGAATATATTCAGATCGATCTGCGCTTTTTTG-3', and the reverse primer is 5'-GATCCAAAAAAGCGCAGATCGATCTGAATATATTCAAGAGATATATTCAGATCGATCTGCGC-3'. Small interfering (si)RNA oligonucleotides were annealed and cloned into the pb7SK/GFP/Neo vector (C02007, GenePharma Corporation, Shanghai, China). The $20-\mu \mathrm{L}$ mixture contained $2 \mu \mathrm{L}$ of annealing buffer, $4 \mu \mathrm{L}$ of sense and antisense siRNA oligonucleotide mix $(100 \mu M)$, and $14 \mu \mathrm{L}$ of double-distilled water.

\section{Constructing Overexpression Vector pBI-CMV3-CD44}

The coding region of $C D 44$ in cow was amplified by PCR with forward primer 5'-ACGCGTATGGACACGTTTTGGTGGC-3' (the underlined sequence indicates a MluI site) and reverse primer 5'-GCGGCCGCTTACACCCCAATCTTCATATCCAC-3' (the underlined sequence indicates a NotI site). The 1,107bp PCR product (MluI to NotI) was validated through DNA sequencing and ligated into the pBI-CMV3 plasmid (\#631632, Clontech Laboratories, Mountain
View, CA) to generate the pBI-CMV3-CD44 expression vector.

\section{Transfection of Plasmids into bMEC}

The bMEC in the study were purified and cultured from a healthy dairy cow according to previous work performed in our laboratory using the method of tissue nubble culture (Lu et al., 2012). Animal experiments were performed in strict accordance with the guidelines for the care and use of laboratory animals by Jilin University (Animal Care and Use Committee permit number: SY201901007). The bMEC were cultured in 10-cm culture plates (353003, Falcon, Franklin Lakes, NJ) in Dulbecco's modified Eagle medium with nutrient mixture F-12 (12-719Q, HyClone, Logan, UT) supplemented with $10 \%$ fetal bovine serum (11011-6123, Tian Hang, Zhejiang, China), $25 \mu \mathrm{L}$ of hydrocortisone (25 $\mu \mathrm{g} / \mathrm{mL}$, ab141250, Abcam, Cambridge, UK), and $25 \mu \mathrm{L}$ of insulin (0.25 EU/mg, I0305000, Sigma-Aldrich, St. Louis, MO) with $1 \%$ antibiotics (SV30010, HyClone). To investigate the regulatory role of CD44 on bMEC, cells were seeded into 6-well culture plates (353090, Falcon) at a concentration of $2 \times 10^{5}$ cells/well and cultured at $37^{\circ} \mathrm{C}$ and $5 \% \mathrm{CO}_{2}$ in a $\mathrm{CO}_{2}$ incubator (Thermo Fisher Scientific, Waltham, MA). When cells reached $80 \%$ confluence, the cell culture medium was changed to a medium without antibiotics for transfection (Jiang et al., 2018). The various vectors were transfected into cells using a FuGENE HD Transfection Reagent according to the manufacturer's protocol (PRE2311, Promega, Madison, WI). Briefly, the mixture of $3 \mu \mathrm{g}$ of plasmid DNA and $7.5 \mu \mathrm{L}$ of FuGENE HD in $150 \mu \mathrm{L}$ of Opti-MEM (Thermo Fisher Scientific) was incubated at room temperature for $15 \mathrm{~min}$ and then added into the culture medium in each well of bMEC. After 24 $\mathrm{h}$, the expression of green fluorescent protein (GFP) in cells was observed under a fluorescence microscope (TE2000, Nikon, Tokyo, Japan) to determine the transfection efficiency. Three replicates of the experiments were carried out by transfecting equal numbers of cells using the same vectors in the various wells.

\section{Analysis of CD44 Expression by Quantitative Real-Time PCR}

Cells transfected with $C D 44$ RNAi or overexpression vector were harvested at $24 \mathrm{~h}$ post-transfection. Then, 1 $\mu \mathrm{g}$ of total RNA was extracted with an RNA extraction kit (Analytik Jena, Jena, Germany), and the concentration and purity of the RNA were determined using a spectrophotometer, further confirmed via $1 \%$ agarose gel electrophoresis. The cDNA was obtained using a 
cDNA synthesis kit (RR047A, TaKaRa Biotechnology, Dalian, China). Then, quantitative real-time (qRT)PCR was performed with SYBR Premix Ex Taq (RR420L, TaKaRa Biotechnology ) with forward primer 5'-CCTCGGATACCAGAGACTACG-3' and reverse primer 5'-CACACCTTCTCCTACTGTTGAC-3'. We performed PCR amplification in $10-\mu \mathrm{L}$ reaction volume under the following conditions: an initial denaturing step of $95^{\circ} \mathrm{C}$ for $30 \mathrm{~s}$, followed by 40 cycles of $95^{\circ} \mathrm{C}$ for $10 \mathrm{~s}$, and then $60^{\circ} \mathrm{C}$ for $45 \mathrm{~s}$. All samples were adjusted to the same concentration. The mRNA expression was measured via qRT-PCR with $\beta$-actin as the reference gene for internal control. All qRT-PCR were repeated at least 3 times.

\section{Determination of CD44 Protein Expression by Western Blotting}

Cells were collected at $48 \mathrm{~h}$ after transfection and were washed 2 to 3 times with PBS (21-040-CVR, Corning Inc., Corning, NY). Cells were then resuspended in radio immunoprecipitation assay buffer (AR0105, Boster Bio, Pleasanton, CA) containing protease and phosphatase inhibitors. Cell lysates were centrifuged at $10,000 \times g$ for 10 min at $4^{\circ} \mathrm{C}$, and the supernatants that contained the target protein were collected. The total protein concentration was measured with the Enhanced BCA Protein Quantitation Assay Kit (KGP902, Keygen Biotech, Jiangsu, China). The 3 repeated experiments on the same sample were performed as technical replicates, and protein concentration was measured in triplicate. A negative control treatment was included, in which double-distilled water was added instead of the protein. Averages were calculated to analyze the contents. Polyvinylidene fluoride membrane was then incubated with rabbit polyclonal anti-CD44 antibody (1:750 dilution in $1 \times$ Tris-buffered saline, bs-4916, Bioss Antibodies, Beijing, China) and $\beta$-actin (1:10,000 dilution in $1 \times$ Tris-buffered saline, ab8227, Abcam, Shanghai, China) for $14 \mathrm{~h}$ at $4^{\circ} \mathrm{C}$. The procedure was performed according to a previously published protocol (Jiang et al., 2018). The relative expressions of the target proteins in different samples were calculated with a gray value analyzer (5200, Tanon Science and Technology Co. Ltd., Shanghai, China).

\section{Analysis of the Triglyceride and Cholesterol Contents in bMEC}

Triglyceride content was measured using a TG detection kit, and CHOL content was determined using a tissue and cell total CHOL assay kit (E1015-105 and E1013, Applygen Technologies, Beijing, China). Cells were seeded at $2 \times 10^{5}$ cells per well in 6 -well culture plates and transfected with different vectors, with triplicates for each vector (353090, Falcon). Cells were collected $48 \mathrm{~h}$ after transfection. A blank control group was set as the reference group using double-distilled water. The cellular contents of TG and CHOL were adjusted by each microgram of protein. The concentrations were measured using a microplate reader (SM600, Shanghai YongChuang Medical Instrument Co. Ltd., Shanghai, China).

\section{FA Extraction and Content Analysis in bMEC by GC}

Fatty acids were extracted from bMEC $48 \mathrm{~h}$ after transfection. Cells were trypsinized after washing 3 times with PBS. Cell pellets were collected through centrifugation. Folch's solution $(\mathrm{CHCl} 3: \mathrm{CH} 3 \mathrm{OH}=2: 1$, $\mathrm{vol} / \mathrm{vol}$ ), an internal reference FA (ginkgolic acid C13:0, 49962, Sigma-Aldrich) was added to cells, and a standard FA (Supelco 37, 18919-1AMP, Sigma-Aldrich) was selected as the standard solution. The tubes with cell pellets were then filled with high-purity nitrogen and shaken vigorously. After evaporation of chloroform, 1 $\mathrm{mL}$ of methyl esterified mixed solvent composed of $35 \%$ BF3 methanol (14\%) (33040-U, Sigma-Aldrich), 45\% methanol, $20 \%$ hexane was added to the glass tubes. Finally, $1 \mathrm{~mL}$ of hexane and $0.4 \mathrm{~mL}$ of $\mathrm{NaCl}(0.88 \%)$ were added, and the supernatant was transferred to clean glass sample bottles with caps via a Pasteur pipette with a long straw for GC analysis (GC7980, ALS7020, Techcomp, Hong Kong, China). Detailed operating conditions were as follows: the column used was an HP-FFAP elastic quartz capillary column (100 $\mathrm{m} \times 0.25 \mathrm{~mm}, 0.2-\mu \mathrm{m}$ film thickness), and the initial column temperature was set at $70^{\circ} \mathrm{C}$. The injection and detector temperatures were $250^{\circ} \mathrm{C}$ and $255^{\circ} \mathrm{C}$, respectively. The split ratio was $1: 1$, and the carrier gas was nitrogen. The injection volume was $1.0 \mu \mathrm{L}$ under operating conditions. The flow rate of hydrogen, nitrogen, and air gases were 25, 20, and $150 \mathrm{~mL} / \mathrm{min}$ at the exit port, respectively. Relative retention time was $119.5 \mathrm{~min}$. Cleaning was conducted after every 4 samples. The contents of FA components were calculated by peak area normalization. The 37 standard FA were measured, and then the emergent times of the 37 FA could be shown. The concentration and the added volumes of the internal reference FA were known, and the contents of the tested FA could be calculated according to the quantity of the internal reference FA and that FA's proportion of total FA, and the proportion of tested FA in total FA. The experiment was repeated 3 times, providing 3 biological replicates, and each replicate was measured 3 times. 


\section{Analysis of Lipid and FA Metabolism by $R T^{2}$ Profiler PCR Array}

One microgram of total RNA was extracted from bMEC with an RNeasy Mini Kit (74134, Qiagen). Then cDNA was synthesized with an $\mathrm{RT}^{2}$ First Strand Kit (330404, Qiagen) according to the manufacturer's protocol. The qRT-PCR was carried out with the Mx3005p system (Stratagene, Agilent, Santa Clara, CA). Transcript levels of related genes in lipid and FA metabolism pathways were determined using an $\mathrm{RT}^{2}$ Profiler PCR Array (CLAB24070A, Qiagen). Five different housekeeping genes were chosen as the reference controls: $\beta$-actin $(A C T B)$, glyceraldehyde-3-phosphate dehydrogenase $(G A P D H)$, tyrosine 3-monooxygenase (YWHAZ), hypoxanthine phosphoribosyltransferase 1 (HPRT1) and TATA-box binding protein (TBP). Threshold count $(\mathbf{C t})$ values were normalized based on the selection of reference genes. The expression fold change or regulation was calculated through a data analysis web portal using the $\Delta \Delta \mathrm{Ct}$ method, in which $\Delta \mathrm{Ct}$ is calculated between the gene of interest and an average of reference genes, followed by $\Delta \Delta \mathrm{Ct}$ calculations $[\Delta \mathrm{Ct}$ (test group) $-\Delta \mathrm{Ct}$ (control group)]. Fold change was then calculated using the $2^{-\Delta \Delta \mathrm{Ct}}$ formula.

\section{Statistical Analysis}

Experimental data are shown as mean \pm standard error of the mean. Statistically significant differences were defined as $P<0.05$. Relative gene expression data from qRT-PCR were analyzed using the comparative Ct method $\left(2^{-\Delta \Delta C t}\right)$. GraphPad Prism 6 software (GraphPad Software Inc., La Jolla, CA) with a 2-tailed (unpaired) $t$-test was used for the data analysis. Relative gene expression data were analyzed using the online $\mathrm{RT}^{2}$ Profiler PCR Array data analysis software (https: //www.qiagen.com/cn/shop-old/data-interpretation -systems/bioinformatics/geneglobe-data-analysis -center/). The negative control was used as the control group. We present RNAi or overexpression of $C D 44$ as relative changes compared with the control.

\section{RESULTS}

\section{Construction of the CD44 Expression Vector}

The RNAi vector pb7SK-shCD44 and overexpression vector pBI-CMV3-CD44 were constructed to examine the function of $C D 44$ on the contents of TG and CHOL (Figure 1A). Plasmid vectors were transfected into bMEC (Figure 1B). The expression of green fluorescence protein was observed in the transfected group, but not in the untransfected group, $24 \mathrm{~h}$ after transfection (Figure 1C).

\section{Analysis of CD44 mRNA Expression}

Expression of $C D 44$ was increased in the pBI-CMV3CD44 overexpression vector group compared with the pBI-CMV3 vector group $(P<0.05$, Figure $2 \mathrm{~A})$. In addition, the mRNA expression in the pb7SK-shCD44 vector-transfected bMEC group was lower compared with the pb7SK/GFP/Neo negative control group ( $P$ $<0.05$, Figure 2B).

\section{Analysis of CD44 Protein Expression}

Western blotting results showed that CD44 protein expression in pBI-CMV3-CD44 group was upregulated relative to that in the pBI-CMV3 group (Figure 2C), and the protein expression in the pb7SK-shCD44 group was downregulated relative to that in $\mathrm{pb} 7 \mathrm{SK} / \mathrm{GFP} /$ Neo negative control group (Figure 2D). The CD44 protein level was expressed as the ratio of CD44 protein to $\beta$-actin.

\section{Effect of CD44 on TG Content}

Levels of TG were higher in bMEC transfected with pBI-CMV3-CD44 than in the control bMEC, which were transfected with the pBI-CMV3 empty vector $(P<0.05$, Figure $3 \mathrm{~A})$. In addition, TG content was lower in bMEC transfected with pb7SK-shCD44 than in the control bMEC, which were transfected only with pb7SK/GFP/Neo empty vector $(P<0.05$, Figure $3 \mathrm{~B})$.

\section{Effect of CD44 on CHOL Content}

Levels of CHOL were detected with a CHOL kit, to examine whether the CD44 gene participated in the CHOL metabolism pathway. The results showed that CHOL content was higher in bMEC transfected with pBI-CMV3-CD44 than in the control group ( $P$ $<0.05$, Figure 3C). Furthermore, CHOL content was lower in bMEC transfected with pb7SK-shCD44 than in the control group, which were transfected with only pb7SK/GFP/Neo empty vector $(P<0.05$, Figure $3 \mathrm{D})$.

\section{Effect of CD44 on FA Content}

The FAME mix of 37 components were measured via GC. The contents of various FA are described in Tables 1 and 2. Concentrations of various FA, such as tridecanoic acid, palmitoleic acid, elaidic acid, stearic acid, linoleic acid, and oleic acid, were lower in the $C D 44$ 
overexpression group compared with the negative control group, whereas the concentrations of $\alpha$-linolenic acid; cis-8,11,14-eicosatrienoic acid, cis-5,8,11,14-eico- satetraenoic acid, palmitic acid, and myristic acid were increased. The concentrations of diverse FA, such as tridecanoic acid, palmitic acid, palmitoleic acid, elaidic

A CD44 gene location: Bos taurus Chromosome 15: 66,454,331-66,541,790.

Coding exons: 9, Transcript length: 2029bps, ORF: 1,107 bp, Translation length: 368 residues.

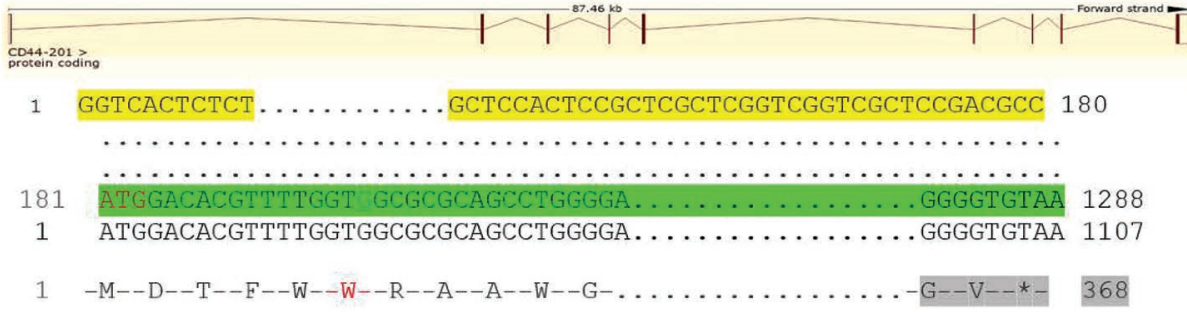

B 1289 САССТАСТTCATGACCTTGGAAATAAАCCACCA..... Forward : 5'-AGAGGCGCAGATCGATCTGAAT (i) ATATCTCTTGAATATATTCAGATCGATCTGCGC (i Forward : 5'-ACGCGTATGGACACGTTTTG TTTTTTG-3'.

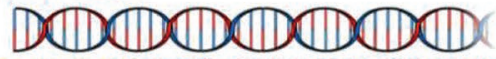

Reverse :5'GATCCAAAAAAGCGCAGATCGAT CTGAATATATTCAAGAGATATATTCAGATCGAT CTGCGC-3'.

C
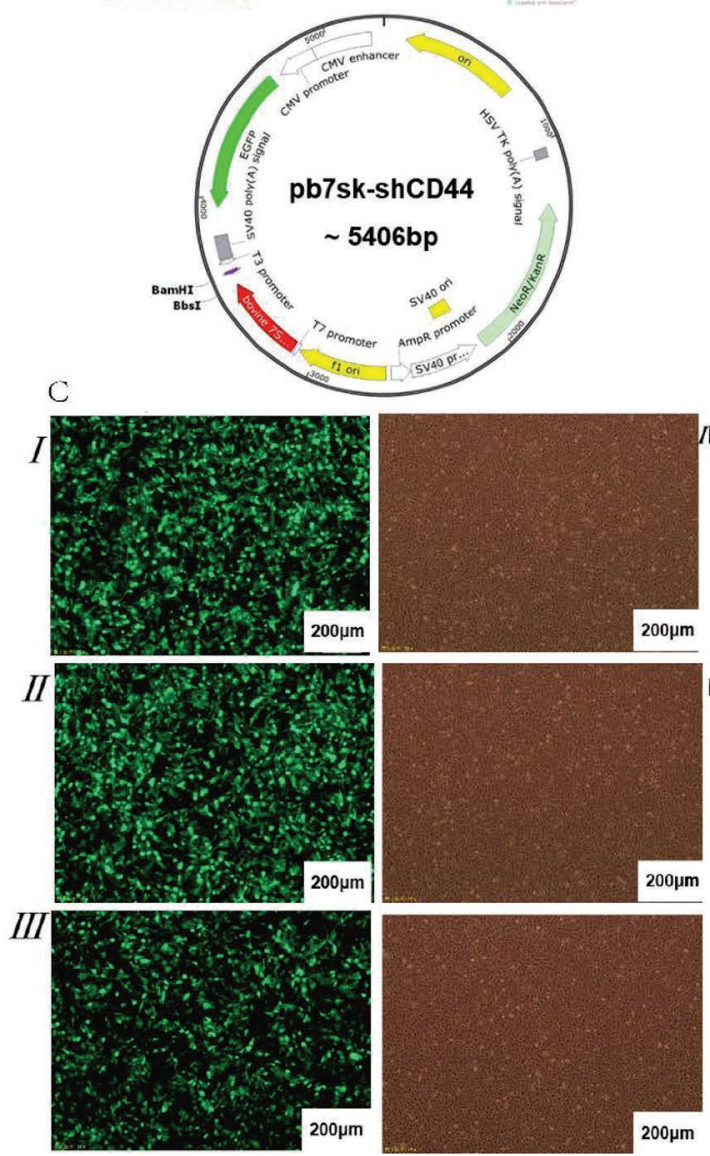

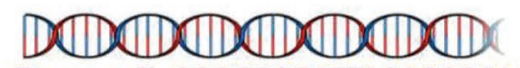
Reverse :5'- GCGGCCGCTTACACCCCAA TCTTCATATCCAC-3،

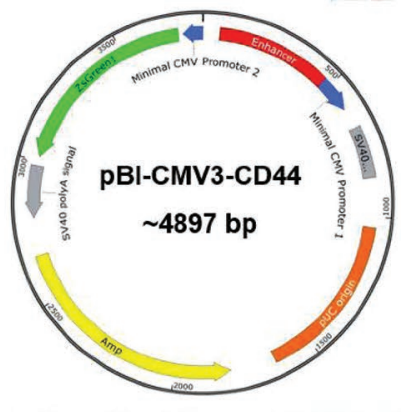

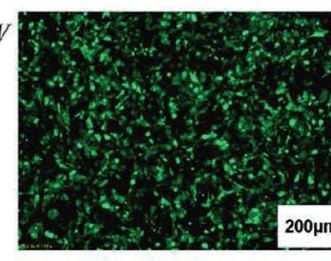
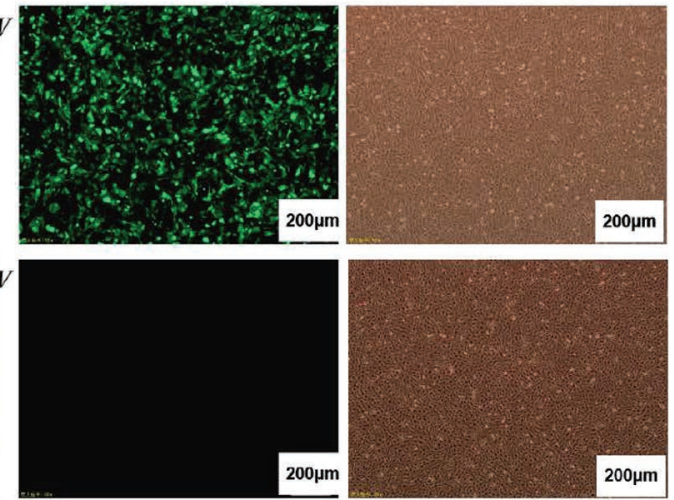

I bMECs transfected with pBICMV3-CD44 II bMECs transfected with pBICMV3 vector III bMECs transfected with pb7Sk-shCD44 $I V$ bMECs transfected with $\mathrm{pb} 7 \mathrm{Sk} / \mathrm{GFP} / \mathrm{Neo}$ $V$ Normal cultured bMECs

Figure 1. Construction of the $C D 44$ gene overexpression vector and the interference vector. (A) The $C D 44$ gene chromosomal location and structural features in bovine. (B) Vector construction for overexpression and knockdown of the CD44 gene. (i) Construction of the $C D 44$ gene interference vector (pb7SK-shCD44). (ii) The construction of the CD44 gene overexpression vector (pBICMV3-CD44). (C) Overexpression and interference vectors transfected into bovine mammary epithelial cells (bMEC). GFP = green fluorescent protein. 
acid, linoleic acid, $\alpha$-linolenic acid, and cis-8,11,14-eicosatrienoic acid were decreased in the $C D 44$ knockdown group. Moreover, the concentrations of stearic acid and cis-5,8,11,14-eicosatetraenoic acid were increased in this group compared with the control group. In addition, oleic acid was not detected in the CD44 knockdown group, which might have resulted from the silencing of CD 44 expression. However, myristic acid was detected in the overexpression group, suggesting that its content may be regulated by the $C D 44$ gene.

\section{Expression of Genes Involved in Lipid Metabolism Pathway}

The results indicated that RNAi of $C D 44$ in bMEC led to upregulation of 4 genes, and the other 4 genes

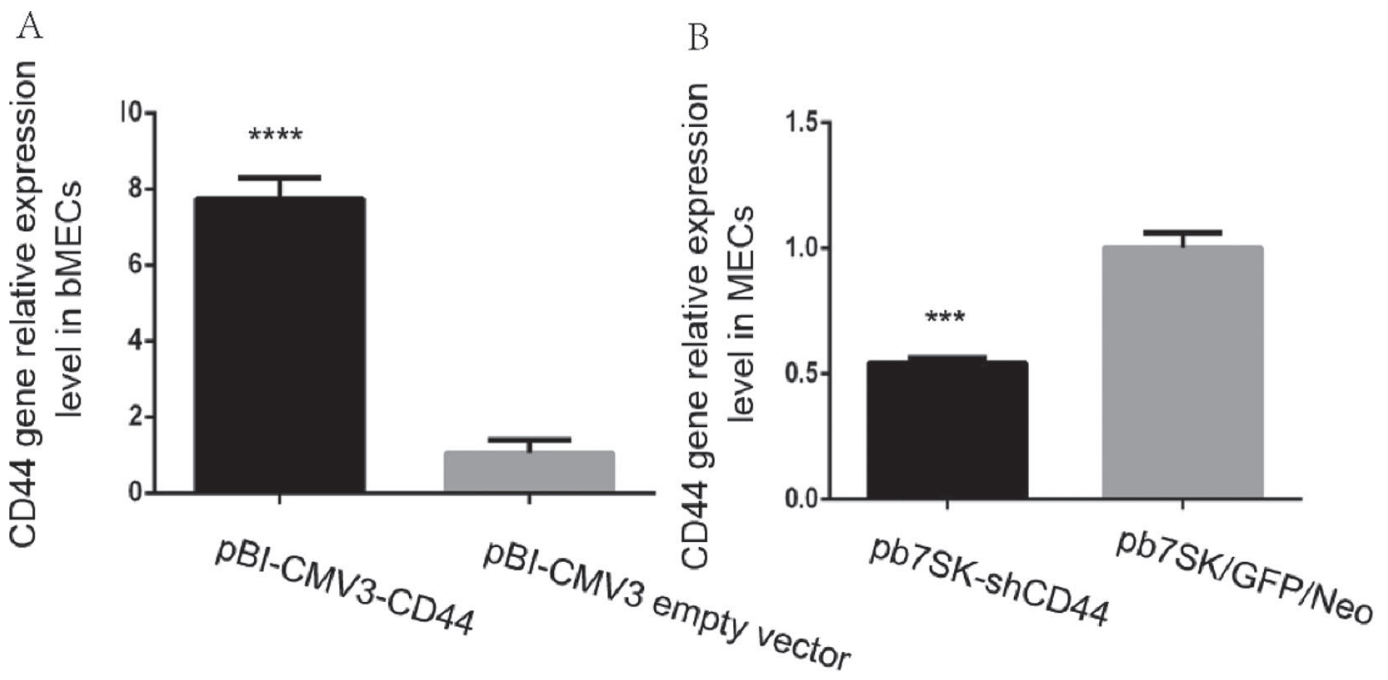

C

D
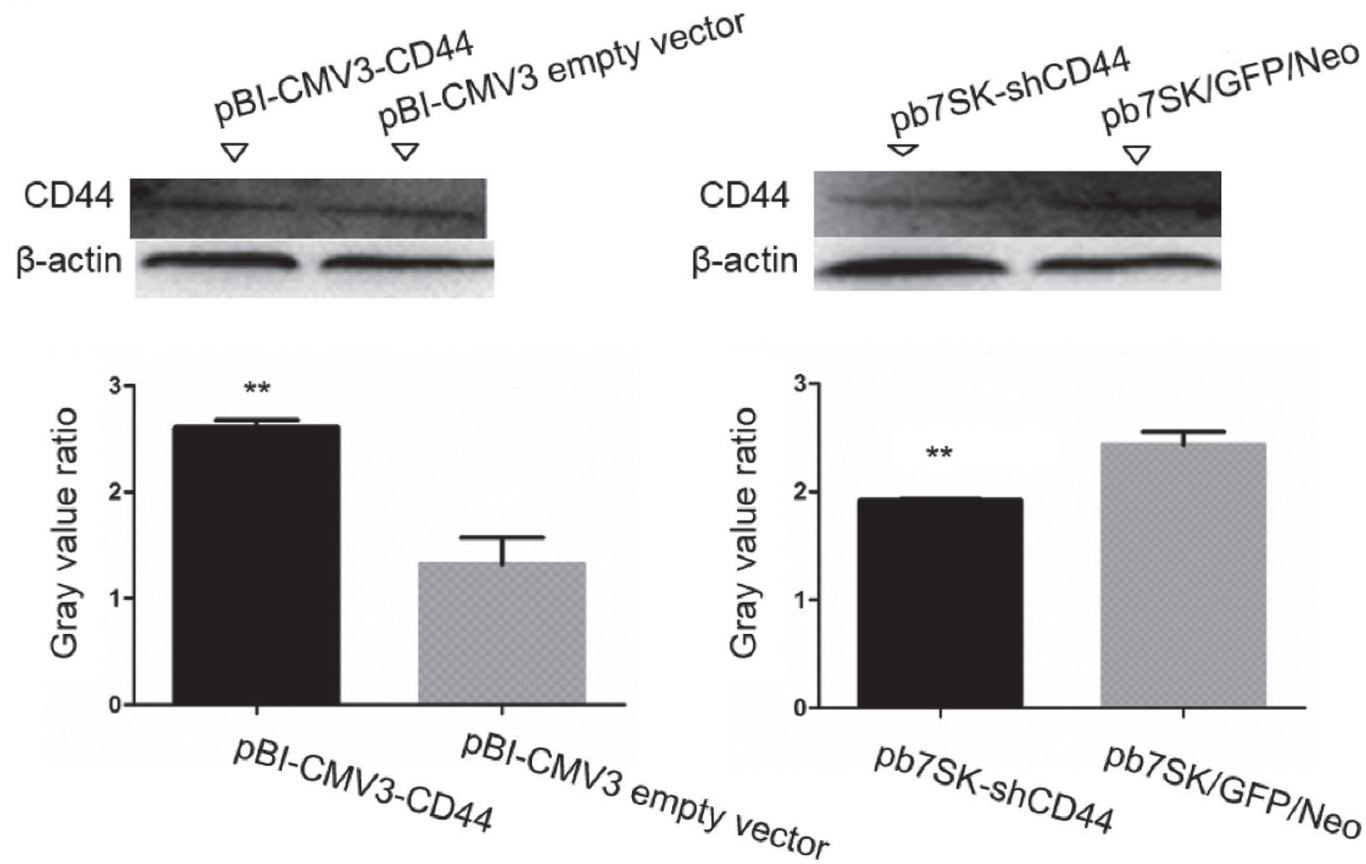

Figure 2. Protein and mRNA expression levels of the $C D 44$ gene in bovine mammary epithelial cells (bMEC). (A) Relative expression levels of $C D 44$ in bMEC transfected with the pBI-CMV3-CD44 vector. (B) Relative expression levels of $C D 44$ in bMEC transfected with the pb7SKshCD44 vector. (C and D) Protein expression levels of CD44 in bMEC transfected with the pBI-CMV3-CD44 and pb7SK-shCD44 vectors. Gray value ratios were calculated as target protein content or $\beta$-actin content. Experimental data are shown as mean \pm SEM. GFP $=$ green fluorescent protein. 
Table 1. The concentrations of various fatty acids in bovine mammary epithelial cells transfected with pBICMV3-CD44 and pBI-CMV3; experimental data are shown as mean \pm SEM

\begin{tabular}{|c|c|c|c|}
\hline Fatty acid component & pBI-CMV3-CD44 & pBI-CMV3 & $P$-value \\
\hline Tridecanoic acid & $0.1471 \pm 0.01279$ & $0.3923 \pm 0.0266$ & 0.0011 \\
\hline Myristic acid & $0.0767 \pm 0.0000$ & 0 & \\
\hline Palmitoleic acid & $0.1471 \pm 0.0128$ & $0.2394 \pm 0.0115$ & 0.0058 \\
\hline Stearic acid & $0.1279 \pm 0.0128$ & $0.1396 \pm 0.0000$ & 0.4102 \\
\hline Elaidic acid & $2.5703 \pm 0.1535$ & $3.3245 \pm 0.0931$ & 0.0137 \\
\hline Oleic acid & $0.2813 \pm 0.0231$ & $0.4056 \pm 0.0290$ & 0.0284 \\
\hline Linoleic acid & $0.3772 \pm 0.0169$ & $0.3923 \pm 0.0266$ & 0.6580 \\
\hline$\alpha$-Linolenic acid & $0.0320 \pm 0.0064$ & $0.01995 \pm 0.000$ & 0.1332 \\
\hline cis-8,11,14-eicosatrienoic acid & $0.3005 \pm 0.0279$ & $0.2074 \pm 0.0925$ & 0.3898 \\
\hline cis-5,8,11,14-eicosatetraenoic acid & $0.1023 \pm 0.0064$ & 0 & \\
\hline Palmitic acid & $6.2404 \pm 0.5427$ & $5.8311 \pm 0.3595$ & 0.5636 \\
\hline
\end{tabular}

A

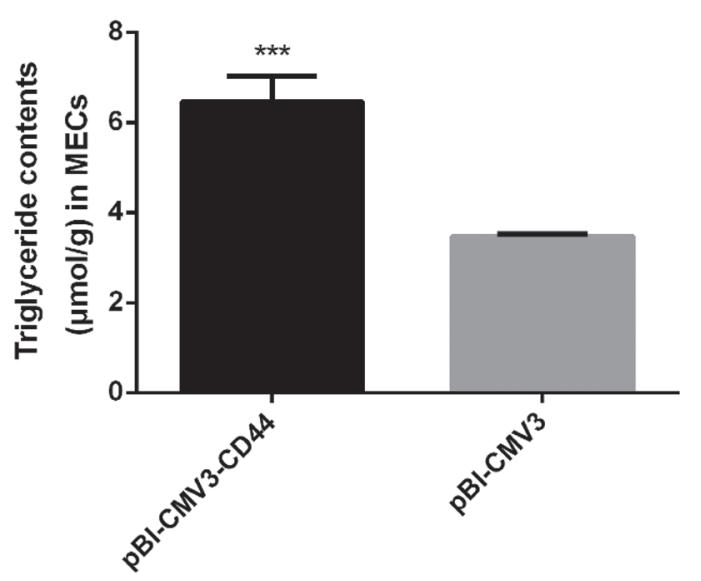

C

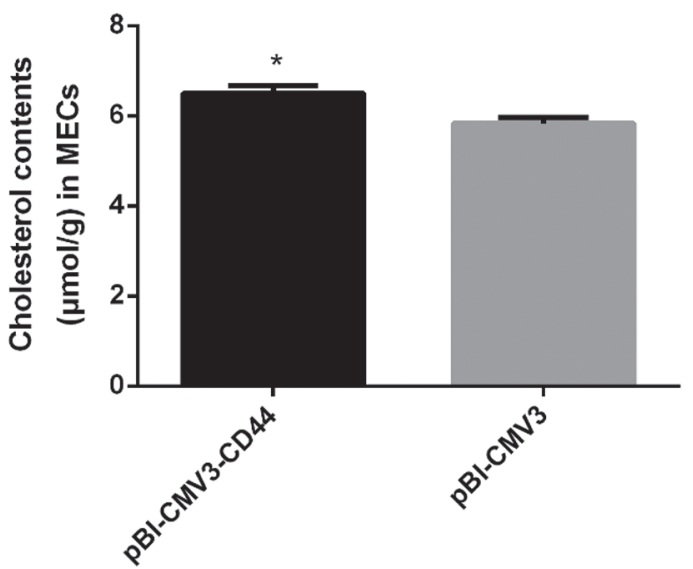

B

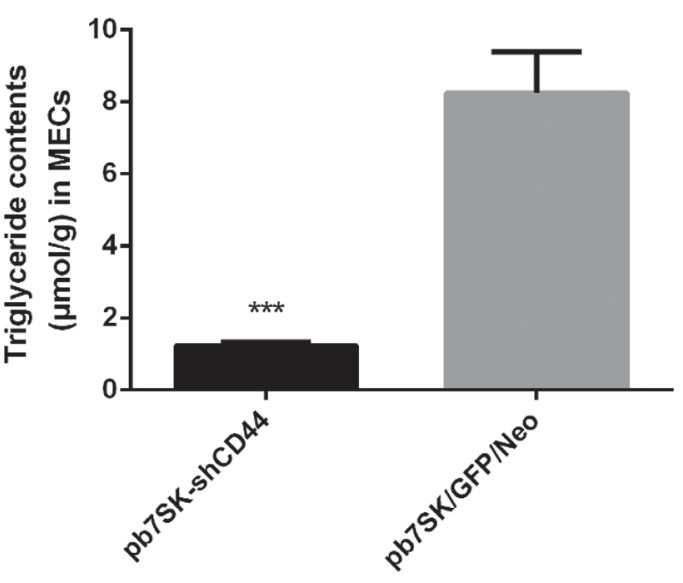

$\mathrm{D}$

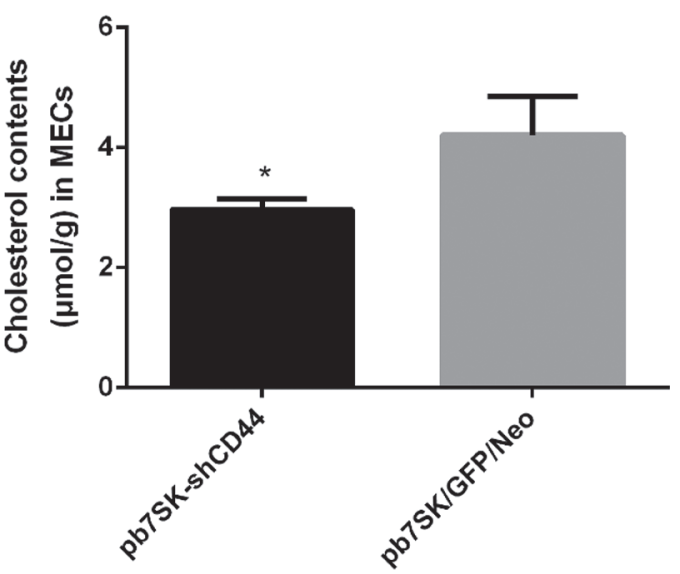

Figure 3. Effects of CD44 on triglyceride (TG) and cholesterol (CHOL) content in bovine mammary epithelial cells (bMEC). (A) TG content in bMEC transfected with the pBI-CMV3-CD44 overexpression vector. (B) TG content in bMEC transfected with the pb7SK-shCD44 vector. (C) CHOL content in bMEC transfected with the pBI-CMV3-CD44 overexpression vector. (D) CHOL content in bMEC transfected with the pb7SK-shCD44 vector. Experimental data are shown as mean $\pm \mathrm{SEM}$. GFP $=$ green fluorescent protein. ${ }^{*} P<0.05 ;{ }^{* * *} P<0.0001$. 
Table 2. The concentrations of various fatty acids are described in bovine mammary epithelial cells transfected with the pb7Sk-shCD44 vector and negative control; experimental data are shown as mean $\pm \mathrm{SEM}$

\begin{tabular}{lccc}
\hline Fatty acid component & pb7SK-shCD44 & Negative control & $P$-value \\
\hline Tridecanoic acid & $0.1661 \pm 0.0315$ & $0.1887 \pm 0.0360$ & 0.6615 \\
Palmitic acid & $5.8566 \pm 0.4477$ & $6.7060 \pm 0.6347$ & 0.3356 \\
Palmitoleic acid & $0.1180 \pm 0.0076$ & $0.1769 \pm 0.0068$ & 0.0044 \\
Stearic acid & $0.2010 \pm 0.0087$ & $0.1494 \pm 0.0079$ & 0.0117 \\
Elaidic acid & $1.5822 \pm 0.7045$ & $2.4135 \pm 0.2039$ & 0.3203 \\
Linoleic acid & $0.2622 \pm 0.0303$ & $0.3695 \pm 0.0283$ & 0.0609 \\
$\alpha$-Linolenic acid & $0.6381 \pm 0.0350$ & $0.7626 \pm 0.0283$ & 0.0506 \\
cis-8,11,14-eicosatrienoic acid & $0.1923 \pm 0.0231$ & $0.2987 \pm 0.0208$ & 0.0267 \\
cis-5,8,11,14-eicosatetraenoic acid & $0.2360 \pm 0.0262$ & $0.1336 \pm 0.0079$ & 0.0201 \\
Oleic acid & 0 & $1.1557 \pm 0.7904$ & \\
\hline
\end{tabular}

were downregulated. Overexpression of $C D 44$ in bMEC upregulated 21 genes and downregulated 23 genes (Tables 3 and 4). All the differentially expressed genes involved in lipid and FA metabolism pathways are presented in Tables 3 and 4 .

\section{DISCUSSION}

The main physiological function of dairy cow mammary tissue is to synthesize and secrete milk. Changes in lipid metabolism can affect numerous cellular processes, including cell growth and differentiation. The biosynthesis and storage of TG are an important cellular process. Cells such as bMEC can accumulate TG in lipid droplets. The successful isolation and culture of bMEC has been widely used in the functional verification of exogenous genes, the mechanism of study of dairy cow mastitis and udder development. Recent studies have showed that different micro (mi)RNA, such as miR-21-3p targets Elovl5 and regulates triglyceride production in bMEC ( $\mathrm{Li}$ et al., 2019); miR-152 regulates triglyceride production in bMEC by targeting the ACAA2 and HSD17B12 genes, which are associated with lipid metabolism (Yang et al., 2018). Altering the expression of these genes could regulate the TG content in bMEC. In addition, the GRK2 gene can negatively regulate milk protein synthesis by activating

Table 3. Fold changes in the expression of genes involved in lipid metabolism that are regulated by $\mathrm{CD} 44$ silencing in bovine mammary epithelial cells

\begin{tabular}{lll}
\hline $\begin{array}{l}\text { Gene } \\
\text { symbol }\end{array}$ & $\begin{array}{c}\text { Fold } \\
\text { change }\end{array}$ & $\begin{array}{l}\text { Reference } \\
\text { sequence }\end{array}$ \\
\hline ACAD11 & 4.1125 & NM_001075893 \\
ACADS & 0.2207 & NM_001034401 \\
ACADSB & 0.6507 & NM_001017933 \\
ACOT9 & 0.0579 & NM_001034560 \\
ACOX2 & 1.7171 & NM_001102015 \\
ACSBG1 & 2.8481 & NM_001024548 \\
ACSBG2 & 0.6285 & NM_174290 \\
ELOVL3 & 1.5801 & NM_001192306 \\
\hline
\end{tabular}

the mitogen-activated protein kinase pathway in bMEC (Hou et al., 2016). Therefore, bMEC can be used as an experimental model to study the function of genes that regulate the synthesis of milk fat components and identify candidate genes related to milk fat synthesis. Triglycerides, CHOL, and FA are the predominant components of milk fat and are recognized as important sources of nutrients in organisms, building blocks for synthesis of cellular components, and energy sources (Lock and Bauman, 2004).

Our previous transcriptome analysis revealed that CD44 is a differentially expressed gene that might be associated with milk fat synthesis. There are certain limitations to this previous model for studying TG synthesis in the mammary gland (Hosseini et al., 2013). Previous studies have shown that bMEC could be used as a cell model to study target genes associated with milk component synthesis (Hou et al., 2016), so we designed our experiment with bMEC to provide information to gain insight into the regulation mechanisms of the $C D 44$ gene, contributing to milk fat synthesis.

The CD 44 gene could regulate the CHOL content of milk. The ATP-binding cassette (ABC) subfamily E member 1 ( $A B C E 1)$ is a key member of the superfamily of $\mathrm{ABC}$ transporters, which is regulated by $C D 44$ and predicted to be associated with $C D 44$ via IID UniProtKB STRING (https://version11.string-db .org/newstring_cgi/show_edge_details.pl?identifiers= 9606.ENSP00000398632\%0D9606.ENSP00000296577) in the present study. At the same time, the ABC family proteins could utilize ATP as a source of energy for transporting lipids and cholesterol across membranes (Dean et al., 2001), which indicates that CD44 might regulate $\mathrm{CHOL}$ by altering the expression of $\mathrm{ABC}$ family proteins.

Fatty acids have different turnover rates in vivo and are either broken down by a series of mitochondrial $\beta$-oxidation processes into acetyl-CoA to contribute to ATP generation through catabolic metabolism or incorporated into triacylglycerol and CHOL esters through 


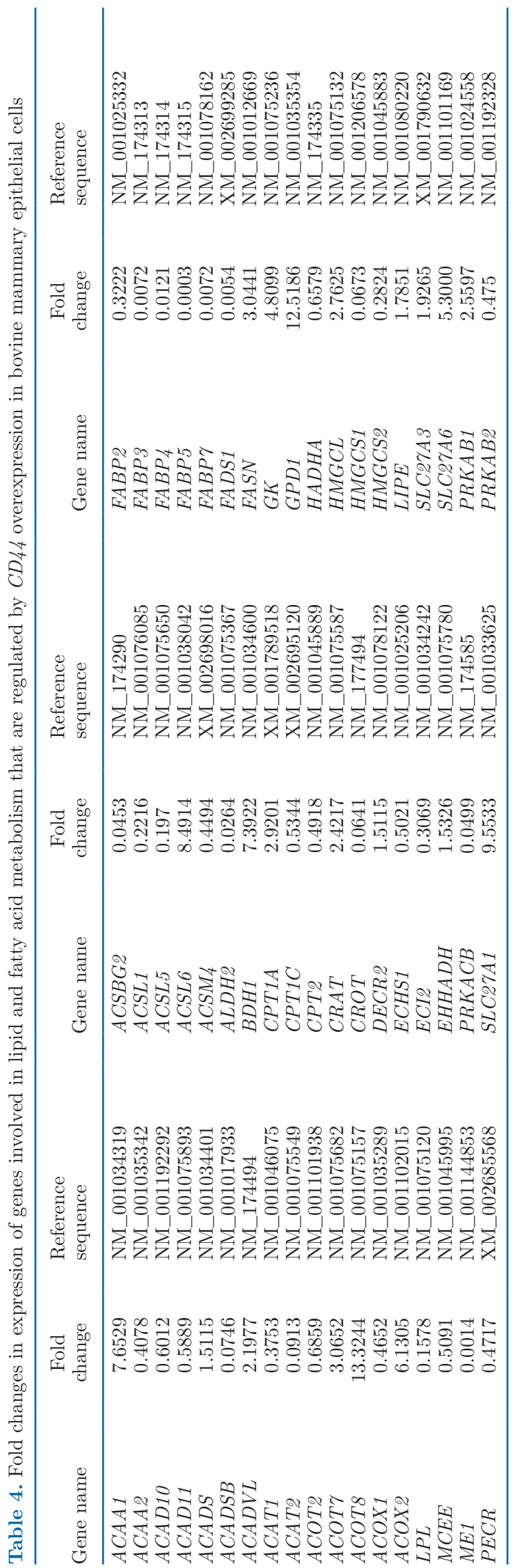

anabolic metabolism (Tang et al., 2018). Overexpression of CD44 in bMEC increased the contents of TG and $\mathrm{CHOL}$, and the FA concentrations of $\alpha$-linolenic acid, cis-8,11,14-eicosatrienoic acid, and palmitic acid were also increased. Furthermore, the inhibition of CD44 expression in bMEC suppressed TG and CHOL contents, and the concentrations of $\alpha$-linolenic acid, cis-8,11,14-eicosatrienoic acid, and palmitic acid were also reduced relative to the negative control group. Remarkably, $\alpha$-linolenic acid, palmitic acid, and cis8,11,14-eicosatrienoic acid were regulated by the expression of CD44. Cis-8,11,14-eicosatrienoic acid, palmitic acid, and elaidic acid are essential for lipid metabolism (Strum-Odin et al., 1987; Schirmer and Phinney, 2007; Ishibashi et al., 2016; Tran et al., 2016; Yang et al., 2016; Montakhab-Yeganeh et al., 2018). Unexpectedly, palmitoleic acid in the $C D 44$ overexpression group was significantly decreased compared with the negative control group, and also decreased in the $C D 44$ knockdown group. We speculated that the palmitoleic acid content in cells might be regulated by other genes or pathways, and the mechanism for palmitoleic acid content decreasing by altered $C D 44$ expression requires further study.

Many protease receptors and transporters are involved in the process of lipid metabolism. These receptors and transporters are regulated by signal transduction pathways to form a complex and delicate regulatory network that maintains the lipid metabolism balance of cells as well as of the whole organism. Previous studies have also indicated that $C D 44$ is a key gene involved in lipogenesis. The present study showed that $C D 44$ is a crucial gene affecting the contents of CHOL, FA, and TG in bMEC. The expression of $C D 44$ significantly regulates genes related to lipid metabolism, such as $A C A D 11$, an important member of the acyl-CoA dehydrogenase family that catalyzes the $\alpha$-, $\beta$-dehydrogenation of acyl-CoA esters using the electron transfer flavoprotein as the physiologic electron acceptor in mitochondria (He et al., 2011), and the expression of $A C A D 11$ was significantly increased by knockdown of the $C D 44$ gene, whereas the expression of $A C A D 11$ was significantly reduced by overexpression of $C D 44$, indicating that $A C A D 11$ is negatively regulated by $C D 44$ gene expression. Acyl-CoA dehydrogenase short chain $(A C A D S)$ is another member of the acylCoA dehydrogenase family and catalyzes the first step in mitochondrial $\beta$-oxidation of FA (He et al., 2011). Interestingly, the expression of $A C A D S$ was significantly reduced by silencing the $C D 44$ gene. Correspondingly, the expression of $A C A D S$ was significantly increased by the overexpression of $C D 44$. This data revealed that $A C A D S$ expression is positively regulated by $C D 44$. The $C D 44$ gene inhibited the expression of $A C A D S B$ and $A C S B G 2$, while promoting the expression of $A C O X 2$. 
The expression level of $A C A D S B$ was positively correlated with cellular TG content and was also actively involved in FA metabolism pathway in bMEC and in milk fat synthesis in dairy cattle (Jiang et al., 2018). These results indicate that $C D 44$ may regulate FA metabolism in bMEC by regulating the expression levels of related genes such as the $A C A D S$ gene.

Carnitine palmitoyltransferase 1A (CPT1A) catalyzes the transfer of acyl of long-chain FA-CoA conjugates onto carnitine, which is a crucial step for mitochondrial uptake of long-chain FA and their subsequent $\beta$-oxidation in mitochondria (Skotte et al., 2017). Carnitine palmitoyltransferase 1C (CPT1C) regulates the $\beta$-oxidation and transport of long-chain FA into mitochondria and play an important role in the regulation of feeding behavior and energy homeostasis. The $C P T 1 C$ related pathways include AMP-activated protein kinase $(A M P K)$ signaling (Wang et al., 2017). Carnitine palmitoyltransferase 2 (CPT2) oxidizes longchain FA in the mitochondria together with carnitine palmitoyltransferase I and is involved in the PPAR- $\alpha$ pathway and metabolism (Lee et al., 2016). Moreover, long-chain acyl-CoA synthetases (ACSL) activate longchain FA (12 to 20 carbons), which are important in FA metabolism (Ehehalt and Füllekrug, 2010; Fuencisla et al., 2011). These results indicate that the expression of $C D 44$ could affect transport and metabolism of FA by up- or down-regulating transcription of related genes and thereby affect the concentration of FA.

In summary, our results showed that $C D 44$ participates in regulating the metabolism of long-chain FA by regulating the expression of related genes involved in FA metabolism pathway. The results demonstrated that $C D 44$ is a key gene that plays a major role in lipid metabolism by regulating the expression of related genes such as $A C A D S$ and $A C A D 11$. The CD44 gene could promote the synthesis of TG and CHOL from FA. This study revealed the function of the $C D 44$ gene on the metabolism of lipid components in vitro in bMEC and identified several key players in the regulatory network of the CD44 gene in FA metabolism, which lays the foundation for further research to unravel the molecular mechanism through which CD44 influences the synthesis of milk fat components in vivo.

\section{ACKNOWLEDGMENTS}

This work was supported by the National R\&D Project of Transgenic Organisms of the Ministry of Science and Technology of China (2016ZX08009003-006; Beijing), the National Natural Science Foundation of China (No. 31772562; Beijing), the Key Platform Project of Innovative Strong School by Education Department of Guangdong Province (ZhanJiang, 2018302), and the Key Laboratory of Animal (Poultry) Genetics Breeding and Reproduction and the Ministry of Agriculture (Poultrylab2018-4; Beijing, China). The authors declare no competing financial interests.

\section{REFERENCES}

Camp, R. L., T. A. Kraus, M. L. Birkeland, and E. Puré. 1991. High levels of CD44 expression distinguish virgin from antigen-primed B cells. J. Exp. Med. 173:763-766. https://doi.org/10.1084/jem 173.3.763.

Dean, M., Y. Hamon, and G. Chimini. 2001. The human ATP-binding cassette (ABC) transporter superfamily. J. Lipid Res. 42:10071017. https://doi.org/10.1101/gr.GR-1649R.

Ehehalt, R., and J. Füllekrug. 2010. CD36, ACSLs and lipid rafts, determinants for cellular fatty acid uptake. Chem. Phys. Lipids 163:S12. https://doi.org/10.1016/j.chemphyslip.2010.05.038.

Fraser, J. K., I. Wulur, Z. Alfonso, and M. H. Hedrick. 2006. Fat tissue: An underappreciated source of stem cells for biotechnology. Trends Biotechnol. 24:150-154. https://doi.org/10.1016/j.tibtech 2006.01.010.

Fuencisla, M. D. B., M. Fedetz, D. Ndagire, A. Alcina Madueño, and M. Sabio, inventors. 2011. Long chain acyl coenzyme-a synthetases (ACSLs) as cyto-serological biomarkers in inflammatory or autoimmune diseases. WO2011/135133. Assignee: Consejo Superior De Investigaciones Científicas (CSIC), Fundación Fibao, Fundación Para La Investigación Biosanitaria De Andalucía Oriental-Alejandro Otero, M. Del Barrio, M. Fedetz, D. Ndagire, A. Alcina Madueño, and M. Sabio.

He, M., Z. Pei, A. W. Mohsen, P. Watkins, G. Murdoch, P. P. Van Veldhoven, R. Ensenauer, and J. Vockley. 2011. Identification and characterization of new long chain Acyl-CoA dehydrogenases. Mol. Genet. Metab. 102:418-429. https://doi.org/10.1016/j.ymgme .2010.12.005.

Hosseini, A., R. Sharma, M. Bionaz, and J. J. Loor. 2013. Transcriptomics comparisons of Mac- $\mathrm{T}$ cells versus mammary tissue during late pregnancy and peak lactation. J. Adv. Dairy Res. 1:103.

Hou, X., H. Hu, Y. Lin, B. Qu, X. Gao, and Q. Li. 2016. The effect of G protein-coupled receptor kinase 2 (GRK2) on lactation and on proliferation of mammary epithelial cells from dairy cows. J. Dairy Sci. 99:5828-5836. https://doi.org/10.3168/jds.2015-10560.

Ishibashi, K., K. Nehashi, T. Oshima, N. Ohkura, and G.-I. Atsumi. 2016. Differentiation with elaidate tends to impair insulin-dependent glucose uptake and GLUT4 translocation in 3T3-L1 adipocytes. Int. J. Food Sci. Nutr. 67:99-110. https://doi.org/10.3109/ 09637486.2016.1144721.

Jensen, R. G., A. M. Ferris, and C. J. Lammi-Keefe. 1991. Symposium: Milk fat-Composition, function, and potential for change: Composition of milk fat. J. Dairy Sci. 74:3228-3243. https://doi.org/10 .3168/jds.S0022-0302(91)78509-3.

Jiang, P., X. Fang, Z. Zhao, X. Yu, B. Sun, H. Yu, and R. Yang. 2018. The effect of short/branched chain acyl-coenzyme A dehydrogenase gene on triglyceride synthesis of bovine mammary epithelial cells. Arch. Tierzucht 61:115-122. https://doi.org/10.5194/aab-61 $-115-2018$.

Kang, H. S., G. Liao, L. M. Degraff, K. Gerrish, C. D. Bortner, S. Garantziotis, and A. M. Jetten. 2013. CD44 plays a critical role in regulating diet-induced adipose inflammation, hepatic steatosis, and insulin resistance. PLoS One 8:e58417. https://doi.org/10 .1371/journal.pone.0058417.

Khan, W. S., A. B. Adesida, S. R. Tew, U. G. Longo, and T. E. Hardingham. 2012. Fat pad-derived mesenchymal stem cells as a potential source for cell-based adipose tissue repair strategies. Cell Prolif. 45:111-120. https://doi.org/10.1111/j.1365-2184.2011.00804.x.

Kodama, K., M. Horikoshi, K. Toda, S. Yamada, K. Hara, J. Irie, M. Sirota, A. A. Morgan, R. Chen, H. Ohtsu, S. Maeda, T. Kadowaki, and A. J. Butte. 2012. Expression-based genome-wide association 
study links the receptor $C D 44$ in adipose tissue with type 2 diabetes. Proc. Natl. Acad. Sci. USA 109:7049-7054. https://doi.org/10 $.1073 /$ pnas.1114513109.

Lee, J., J. Choi, S. Aja, S. Scafidi, and M. J. Wolfgang. 2016. Loss of adipose fatty acid oxidation does not potentiate obesity at thermoneutrality. Cell Rep. 14:1308-1316. https://doi.org/10.1016/j .celrep.2016.01.029.

Li, X., P. Jiang, H. Yu, Y. Yang, L. Xia, R. Yang, X. Fang, and Z. Zhao. 2019. miR-21-3p targets Elovl5 and regulates triglyceride production in mammary epithelial cells of cow. DNA Cell Biol. 38:352-357. https://doi.org/10.1089/dna.2018.4409.

Lock, A. L., and D. E. Bauman. 2004. Modifying milk fat composition of dairy cows to enhance fatty acids beneficial to human health. Lipids 39:1197-1206. https://doi.org/10.1007/s11745-004-1348-6.

Lu, C., R. Yang, B. Liu, Z. Li, B. Shen, S. Yan, Y. Zhang, L. Zhang, and Z. Zhao. 2012. Establishment of two types of mammary epithelial cell lines from Chinese Holstein dairy cow. J. Anim. Vet. Adv. 11:1166-1172. https://doi.org/10.3923/javaa.2012.1166.1172.

Lubary, M., G. W. Hofland, and J. H. Ter Horst. 2011. The potential of milk fat for the synthesis of valuable derivatives. Eur. Food Res. Technol. 232:1-8. https://doi.org/10.1007/s00217-010-1387-3.

Montakhab-Yeganeh, H., H. Babaahmadi-Rezaei, and M. Doosti. 2018. Effect of elaidic acid on ABCA1 expression in raw 264.7 cells. Is it through PPAR-gamma? EXCLI J. 17:864-870.

Schirmer, M. A., and S. D. Phinney. 2007. $\gamma$-Linolenate reduces weight regain in formerly obese humans. J. Nutr. 137:1430-1435. https:// doi.org/10.1093/jn/137.6.1430.

Schmits, R., J. Filmus, N. Gerwin, G. Senaldi, F. Kiefer, T. Kundig, A. Wakeham, A. Shahinian, C. Catzavelos, J. Rak, C. Furlonger, A. Zakarian, J. J. L. Simard, P. S. Ohashi, C. J. Paige, J. C. Gutierrez-Ramos, and T. W. Mak. 1997. CD44 regulates hematopoietic progenitor distribution, granuloma formation, and tumorigenicity. Blood 90:2217-2233. https://doi.org/10.1182/blood.V90.6.2217.

Skotte, L., A. Koch, V. Yakimov, S. Zhou, B. Søborg, M. Andersson, S. W. Michelsen, J. E. Navne, J. M. Mistry, P. A. Dion, M. L. Pedersen, M. L. Børresen, G. A. Rouleau, F. Geller, M. Melbye, and B. Feenstra. 2017. CPT1A missense mutation associated with fatty acid metabolism and reduced height in Greenlanders. Circ. Cardiovasc. Genet. 10:e001618. https://doi.org/10.1161/ CIRCGENETICS.116.001618.

Strum-Odin, R., B. Adkins-Finke, W. L. Blake, S. D. Phinney, and S. D. Clarke. 1987. Modification of fatty acid composition of mem- brane phospholipid in hepatocyte monolayer with $n-3, n-6$ and $n-9$ fatty acids and its relationship to triacylglycerol production. Biochim. Biophys. Acta 921:378-391. https://doi.org/10.1016/0005 $-2760(87) 90040-3$

Tang, Y., J. Zhou, S. C. Hooi, Y. M. Jiang, and G. D. Lu. 2018. Fatty acid activation in carcinogenesis and cancer development: Essential roles of long-chain acyl-CoA synthetases. Oncol. Lett. 16:1390. https://doi.org/10.3892/ol.2018.8843.

Tran, D. Q., E. H. Ramos, and D. D. Belsham. 2016. Induction of Gnrh mRNA expression by the $\omega-3$ polyunsaturated fatty acid docosahexaenoic acid and the saturated fatty acid palmitate in a GnRH-synthesizing neuronal cell model, mHypoA-GnRH/GFP. Mol. Cell. Endocrinol. 426:125-135. https://doi.org/10.1016/j.mce .2016.02.019.

Wang, R., Y. Cheng, D. Su, B. Gong, X. He, X. Zhou, Z. Pang, L. Cheng, Y. Chen, and Z. Yao. 2017. Cpt1c regulated by AMPK promotes papillary thyroid carcinomas cells survival under metabolic stress conditions. J. Cancer 8:3675-3681. https://doi.org/10 $.7150 /$ jca. 21148 .

Yang, W.-M., K.-H. Min, and W. Lee. 2016. MiR-1271 upregulated by saturated fatty acid palmitate provokes impaired insulin signaling by repressing INSR and IRS-1 expression in HepG2 cells. Biochem. Biophys. Res. Commun. 478:1786-1791. https://doi.org/10.1016/ j.bbrc.2016.09.029.

Yang, Y., X. Fang, R. Yang, H. Yu, P. Jiang, B. Sun, and Z. Zhao. 2018. MiR-152 regulates apoptosis and triglyceride production in MECs via targeting ACAA2 and HSD17B12 genes. Sci. Rep. 8:417. https://doi.org/10.1038/s41598-017-18804-x.

\section{ORCIDS}

Ping Jiang $\odot$ https://orcid.org/0000-0001-7341-9070 Lixin Xia @ https://orcid.org/0000-0001-7712-4851 Mengyan Wang @ https://orcid.org/0000-0002-1157-0047 Runjun Yang (๑) https://orcid.org/0000-0003-1401-4318 Xibi Fang ๑ https://orcid.org/0000-0003-1134-0003 Zhihui Zhao @ https://orcid.org/0000-0001-5907-0669 